\title{
Efficiency of Using Mastication Test with Food Stimuli While Performing Electromyographic Studies in Dental Patients
}

\author{
Oksana Kinash*, Mycola Rozhko, Andryyi Kostyshyn
}

\begin{abstract}
The relevance of the topic is related to the necessity for a more detailed study of the effectiveness of surface electromyography as a method for diagnosis of pathological conditions of the teeth-and-jaws system and estimation of the orthopaedic treatment results. The objective of the study was to investigate the effectiveness of using a mastication test while performing surface electromyography in dental patients. We studied and analysed the output of surface electromyograms of m.masseter and m.temporalis obtained from a functional test of the maximum jaw clenching and a mastication test with a food stimulus in 54 persons. The results of the study showed a slight difference in the absolute values between the data of the functional test of the maximum jaw clenching and the mastication test. However, the comparative data of the tests after editing (removal of fragments representing the maximal mouth opening during the mastication from electromyograms in the "Neuro-MSP" program) showed no significant difference in data $(>0.05)$ and the maximum approximation of the absolute values of the functional and mastication tests in all cases. Thus, it has been established that in practice the mastication test while performing electromyography in dental patients should be determined and carried out as an additional one in order to clarify the results of treatment. Further studying and establishment of the most effective methods for electromyography is considered to be promising in dentistry.
\end{abstract}

Keywords

surface electromyography; dentistry; EMG study

Ivano-Frankivsk National Medical University, Ivano-Frankivsk, Ukraine

*Corresponding author: danakinash@gmail.com

\section{Problem statement and analysis of the recent research}

Today electromyography in dentistry is one of the most variable research methods in terms of effectiveness and interpretation of results [1]. One of the stages while performing an electromyography is recording the mastication muscles parameters during a mastication test with a food stimulus (a walnut, a piece of bread or a carrot, a chewing gum) [2, 3, 4]. However, the absence of a unified scheme for carrying out this test and the lack of information about it in foreign sources requires studying its efficacy.

Objective. To investigate the effectiveness of using a mastication test while performing surface electromyography in dental patients.

\section{Materials and methods of the research}

To achieve the objective, we studied and analysed the results of m.masseter and m.temporalis surface EMG during the mastication test in 54 patients as well as the maximum amplitudes of m.masseter and m.temporalis in the state of the maximum jaw clenching in the same patients from the department database.
These researches were performed under the protocol [5], according to which the following tests were carried out: a functional test - recording an electromyogram for $3.5 \mathrm{sec}$ onds during the maximum jaw clenching; a mastication test - recording a mastication cycle of chewing a $0.8 \mathrm{~g}$ walnut. Each test was performed three times. The analysis took into account the mean value of the three equivalent tests.

Considering "Neuro-EMG-Micro" is a two-channel electroneuromyograph, after the muscles of mastication had been examined, the electrodes were positioned on the anterior bundles of the temporal muscles and a corresponding examination of m.temporalis was performed with a 5 minute interval for a patient to rest.

Bioelectric activity of mastication muscles was studied simultaneously in symmetrical muscles on both sides.

The results of the functional test were not further edited, whereas the results of the mastication test were edited in the following way: since the mastication cycle consists of mouth opening when the muscles of mastication are relaxed and the jaw clenching which involves muscles contraction, we deleted the mastication cycle fragments representing the maximum mouth opening from the "Neuro-MSP" program, and the attention was paid to the fragments showing the maximum jaw 
clenching during the mastication cycle with a total length of 3.5 seconds.

\section{Results of the research and their discussion}

In the process of research, the following data the following data have been ascertained.

The results of the conducted study showed a slight difference in the absolute values between the data of the functional test of the maximum jaw clenching and the mastication test with a food stimulus, which in that case was a $0.8 \mathrm{~g}$ piece of walnut, without editing the electromyogram which was the closest in the left m.masseter value - (1292.84 \pm 22.88$)$ $\mu \mathrm{V}$ to $(1193.9 \pm 60.45) \mu \mathrm{V}$, and the furthest in the right m.temporalis value - (1335.84 \pm 43.39$) \mu \mathrm{V}$ up to $(901.81$ $\pm 27.35) \mu \mathrm{V}$, which proves once again the pattern of the interconnection between the cross-sectional m.masseter and $\mathrm{m}$. temporalis.

However, the comparative data of the functional test and the mastication test after editing in all cases revealed no significant difference in data $(>0.05)$ and the maximal approximation of the absolute values of the functional and mastication tests that could especially be observed in the left m.masseter value - (1292.84 \pm 22.88$) \mu \mathrm{V}$ up to $(1285.85 \pm 62.44) \mu \mathrm{V}$. These measurements have shown that there is no fundamental difference between the values of the tests. The reduction in the absolute values is the result of the physiological processes of mastication.

\section{Conclusions}

From the above we can conclude that the mastication test with a food stimulus while performing surface electromyography in dentistry should be applied as a supplementary one in order to clarify the results. After all, the maximum amplitudes values of musculus masseter and musculus temporalis during this test, without taking into account the EMG fragments showing muscle relaxation during the mouth opening in the mastication cycle, have no reliable probability of data with the parameters of the functional test of the maximum law clenching. Also, there is no significant difference when comparing the absolute values of two tests.

\section{Prospects for further research}

Further studying and establishment of the most effective methods for electromyographic study is considered to be promising in dentistry.

\section{References}

[1] Kostyshyn AB, Rozhko MM, Pelelhan LI. Electromyography in Ukrainian orthopedic stomatology: achievements, problems, prospects. Archive of Clinical Medicine. 2011; 2(17):4-6.
[2] Pokrovskyi MM, Khalavka MM, Kuziv SP et al. Functional state of chewing muscles at the stages of complex treatment of periodontitis. Galician Medical Journal. 2003; 10(4):69-72.

[3] Semenenko YuI. Studying the dependence of various factors during electromyographic research on the quality of obtaining results. Ukrainian Dental Almanac. 2010; 4:63.

[4] Kostyshyn AB. The peculiarities of the surface electromyography in young people. Galician Medical Journal. 2014; 21(3):29-31.

[5] Kostyshyn AB, Rozhko MM, Pelelhan LI. Protocol of the EMG-examination of the m.masseter and the m.temporalis in bite height reduced patients by electroneuromyograph "Neuro-EMG-Micro". Precarpathian bulletin of the Shevchenko Scientific Society. Pulse. 2012; 4:49-54.

Received: 6 Apr 2018

Revised: 17 May 2018

Accepted: 22 May 2018 
Table 1. Comparative Characteristic of the Maximum Amplitudes Values $(\mu \mathrm{V})$ of Surface Electromyograms While Performing Functional and Mastication Tests

\begin{tabular}{|c|c|c|c|c|c|c|c|c|}
\hline \multirow[t]{2}{*}{ Muscle } & \multirow[t]{2}{*}{ Side } & \multicolumn{2}{|c|}{$\begin{array}{l}\text { Functional test } \\
\qquad(\mathrm{n}=54)\end{array}$} & \multicolumn{2}{|c|}{$\begin{array}{c}\text { Mastication test } \\
\text { without editing }(n=54)\end{array}$} & \multicolumn{2}{|c|}{$\begin{array}{l}\text { Mastication test } \\
\text { after editing }(n=54)\end{array}$} & \multirow[t]{2}{*}{ p 1-3 } \\
\hline & & Value & $\mathrm{M} \pm \mathrm{m}$ & Value & $\mathrm{M} \pm \mathrm{m}$ & Value & $\mathrm{M} \pm \mathrm{m}$ & \\
\hline \multirow[t]{2}{*}{ m.masseter } & Right & 1335.84 & 43.39 & 1172.7 & 60.12 & 1299.54 & 61.37 & $>0.05$ \\
\hline & Left & 1292.84 & 22.88 & 1193.9 & 60.45 & 1285.85 & 62.44 & $>0.05$ \\
\hline \multirow[t]{2}{*}{ m.temporalis } & Right & 1335.84 & 43.39 & 901.81 & 27.35 & 1241.24 & 37.33 & $>0.05$ \\
\hline & Left & 1292.84 & 22.88 & 917.78 & 33.49 & 1138.68 & 38.15 & $>0.05$ \\
\hline
\end{tabular}

